\title{
Review \\ Efficacy of a Multi-Strain Probiotic Formulation in Pediatric Populations: A Comprehensive Review of Clinical Studies
}

\author{
Annie Tremblay ${ }^{1}$, Xiaoyu Xu ${ }^{1}$, James Colee ${ }^{2}$ and Thomas A. Tompkins ${ }^{1, *(1)}$ \\ 1 Rosell Institute for Microbiome and Probiotics, Lallemand Health Solutions Inc., 6100 Royalmount Avenue, \\ Montreal, QC H4P2R2, Canada; atremblay@lallemand.com (A.T.); jadecher@hotmail.com (X.X.) \\ 2 IFAS Statistical Consulting Unit, University of Florida, P.O. Box 110500, Gainesville, FL 32611-0500, USA; \\ jcolee@gmail.com \\ * Correspondence: ttompkins@lallemand.com
}

check for

updates

Citation: Tremblay, A.; Xu, X.; Colee,

J.; Tompkins, T.A. Efficacy of a

Multi-Strain Probiotic Formulation in

Pediatric Populations: A

Comprehensive Review of Clinical

Studies. Nutrients 2021, 13, 1908.

https://doi.org/10.3390/nu13061908

Academic Editors: Francisco

J. Pérez-Cano and

Miguel Romero-Pérez

Received: 7 May 2021

Accepted: 27 May 2021

Published: 1 June 2021

Publisher's Note: MDPI stays neutral with regard to jurisdictional claims in published maps and institutional affiliations.

Copyright: (c) 2021 by the authors. Licensee MDPI, Basel, Switzerland. This article is an open access article distributed under the terms and conditions of the Creative Commons Attribution (CC BY) license (https:/ / creativecommons.org/licenses/by/ $4.0 /)$.

\begin{abstract}
A probiotic formulation combining Lactobacillus helveticus Rosell ${ }^{\circledR}-52$, Bifidobacterium infantis Rosell $^{\circledR}-33$, and Bifidobacterium bifidum Rosell ${ }^{\circledR}-71$ with fructooligosaccharides, first commercialized in China, has been sold in over 28 countries since 2002. Clinical studies with this blend of strains were conducted mainly in pediatric populations, and most were published in non-English journals. This comprehensive review summarizes the clinical studies in infants and children to evaluate the efficacy of this probiotic for pediatric indications. Literature searches for pediatric studies on Biostime ${ }^{\circledR}$ or Probiokid ${ }^{\circledR}$ (non-commercial name) in 6 international and Chinese databases identified 28 studies, which were classified by indications. Twelve studies show that the probiotic significantly increases the efficacy of standard diarrhea treatment regardless of etiology, reducing the risk of unresolved diarrhea (RR 0.31 [0.23; 0.42]; $p<0.0001$ ) by $69 \%$. In eight studies, the probiotic enhanced immune defenses, assessed by levels of various immune competence and mucosal immunity markers (six studies), and reduced the incidence of common infections (two studies). The probiotic improved iron deficiency anemia treatment efficacy (three studies), reducing the risk of unresolved anemia by $49 \%$ (RR 0.51 [0.28; 0.92]; $p=0.0263$ ) and significantly reducing treatment side effects by $47 \%$ (RR 0.53 [0.37; 0.77]; $p=0.0009$ ). Other studies support further investigation into this probiotic for oral candidiasis, eczema, feeding intolerance in premature babies, or hyperbilirubinemia in newborns.
\end{abstract}

Keywords: probiotics; Biostime ${ }^{\circledR}$; Probiokid ${ }^{\circledR}$; infants; newborns; children; infantile diarrhea; immune defenses

\section{Introduction}

Lactobacilli and Bifidobacteria are commensal bacteria of the human and animal gastrointestinal (GI) tract, and several strains of these genera are widely used as probiotics and in food supplements, or as starter or adjunct cultures in the production of dairy products or other fermented foods. Although Lactobacilli have been shown to represent a small proportion of the fully developed and highly diverse adult microbiota, their importance is demonstrated by the association between their modulation and various diseases [1]. Bifidobacteria were shown to be among the first colonizers of the healthy infant's GI tract, and to predominate in the intestinal tract until the transition to a solid food diet, at which point microbiota diversity begins to increase towards the varied composition typically seen in adults [2]. With the increasing awareness of the role of the microbiota and dysbiosis in pediatric health and diseases [3], the use of probiotics is considered among the potential interventions available to target the microbiota in pediatric populations. However, assessing the overall safety and efficacy of probiotics in general for a given indication increases heterogeneity when the analyses combine studies having assessed a variety of probiotic strains or formulations, dosing regimen, and outcome assessment measures [4].

The probiotic formulation reviewed herein is composed of Lactobacillus (L.) helveticus Rosell ${ }^{\circledR}-52$, Bifidobacterium (B.) longum subsp. infantis (B. infantis) Rosell ${ }^{\circledR}-33$ and B. bifidum 
Rosell ${ }^{\circledR}-71$, with fructooligosaccharides. All three strains were deposited at the Pasteur Institute in the "Collection Nationale de Cultures de Microorganismes" (CNCM), under the numbers CNCM I-1722, CNCM I-3424 and CNCM I-3426, respectively [5,6]. The whole-genome sequences are deposited in the PATRIC database (https:/ /www.patricbrc.org/ (accessed on 31 May 2021)) under the genome identification numbers (Genome ID): 880633.7, 1678.111, and 1678.107, respectively. First marketed in China in 2002, this formulation has since been commercially available in over 28 countries. The innocuity of the included strains is recognized by several authoritative bodies worldwide, where they are included in lists of safe strains for consumption in foods. Probiokid ${ }^{\circledR}$ and the individual strains have been recognized for their safety by the US FDA in the form of a no question letter for notified GRAS status for use in non-exempt infant formula, in Canada by the NNHPD for use in Natural Health Products in infants 3 months old and over, and in China for safe use in food for infants and young children. In addition, the safety of this product is also monitored through a pharmacovigilance program covering both foreign and domestic adverse event cases [7].

The clinical studies conducted with this probiotic formulation have demonstrated its beneficial effects on GI and immune functions in children. With many of the studies published in non-English journals, there is a barrier for results dissemination to the global scientific community. Hence, we undertook this comprehensive literature review summarizing the clinical studies on this probiotic formulation published in North America, Europe, and Asia to provide an overview of the global evidence base available on this probiotic in pediatric populations. In addition, when possible, outcome-specific meta-analyses were conducted on studies harboring similar design and outcome assessment methods, which strengthens the conclusions obtained from individual trials while providing more specificity than the meta-analyses including different probiotic strains or formulations.

\section{Materials and Methods}

The results of this comprehensive review are reported according to the PRISMA guidelines. The protocol of this review was not prospectively registered. Articles of interest were identified by searching for the trade names, Probiokid ${ }^{\circledR}$ (non-commercial name) or Biostime $^{\circledR}\left(\right.$ 合生元 $\left.{ }^{\circledR}\right)$, and for the individual strain names and numbers (Supplemental Table S1) using search engines for scientific or medical journal databases up to May 2021. Two independent assessors conducted the searches, screening against inclusion/exclusion criteria, and data extraction (A.T., X.X.). Discrepancies were solved via discussion between the two authors, and a third assessor was consulted as needed for resolution (T.A.T.). Articles in Chinese, although most had an abstract in English, were translated using Google translate and a native speaker (X.X.) performed inclusion/exclusion criteria assessment and data extraction using the original language articles. For the selection process, retrieved articles were screened by title and abstract to identify the clinical trials in pediatric populations and then examined for study design, regardless of the indication. Only studies where controls allowed for a direct assessment of the effect of the probiotic were included. These encompassed studies where the probiotic formulation was used as adjuvant with the active comparator alone used as control, studies comparing with a placebo, another probiotic (e.g., GoldenBifido, Lacidophilin), or a standard of care (e.g., Smecta ${ }^{\circledR}$, iron dextran, phototherapy) as control, or case-control-type studies allowing for a comparison of the effect before and after the probiotic intervention. Study authors were not contacted to provide additional data.

A total of 28 studies in pediatric populations were included and are detailed in this comprehensive review (Figure 1). 


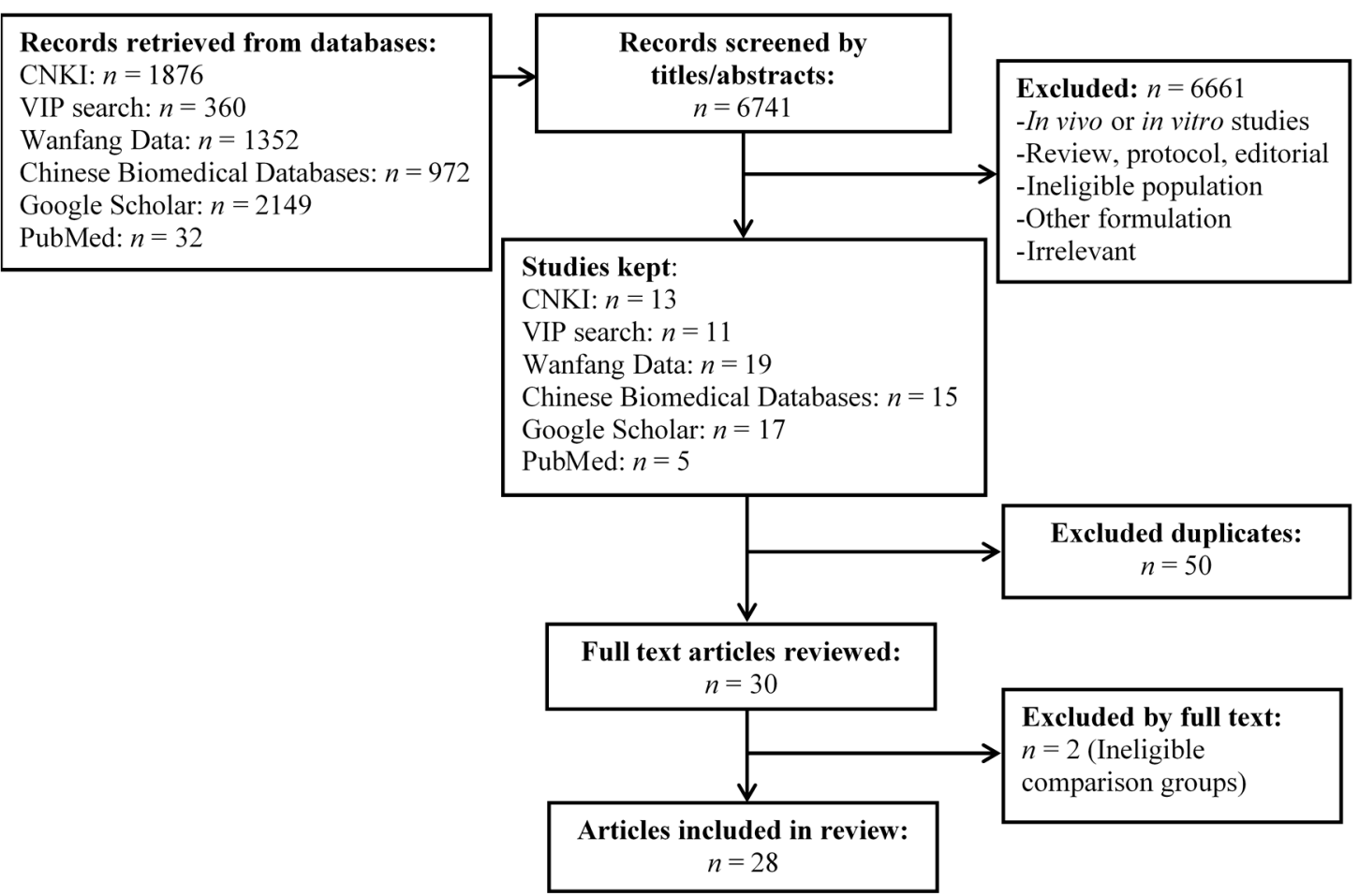

Figure 1. PRISMA flow diagram.

The characteristics of the 28 studies were extracted using a tabular format including study dates, population, study design, arms and $n$, treatment regimen, results, and adverse events (including serious adverse events). All studies were conducted with the same formulation which contains the strains $L$. helveticus Rosell ${ }^{\circledR}-52, B$. infantis Rosell ${ }^{\circledR}-33$ and $B$. bifidum Rosell $^{\circledR}-71$, with 750 mg of fructooligosaccharrides per sachet. This formulation is manufactured to ensure a minimal active dose of 3 billion colony-forming units (CFU) per sachet at the end of shelf-life. Considering that reporting standards for the dosage vary between countries, the Chinese or some European studies tend to report the dose at manufacturing, which is higher to maintain the guaranteed minimal active dose per sachet until the end of shelf-life. Therefore, for comparability, dosage information is provided herein based on the number of sachets administered.

After examination of the study characteristics tables, a meta-analysis was conducted if 3 studies or more allowed to calculate the relative risk for a given outcome when similar design, assessment method and data reporting format were used. Meta-analyses were conducted in $\mathrm{R}$ version 4.0.2, using the meta package [8,9]. Weighing was calculated using the inverse variance method, the DerSimonian-Laird estimator was used for $\tau^{2}$, and the Jackson method was used for confidence interval of $\tau^{2}$ and $\tau$. Studies included in metaanalyses were assessed by 2 authors (A.T., X.X.) for the risk of bias (low, unclear, high) [10], and the overall level of bias was assigned for the treatment efficacy outcome used in the meta-analysis. Discrepancies were resolved through discussion, and a third assessor (T.T.) was consulted as needed. For the studies included in the diarrhea outcome meta-analysis, publication bias was estimated using a funnel plot and asymmetry quantification using the Egger's regression test. The Trim and Fill method by Duval and Tweedie was used to estimate the number of missing studies and these estimated negative studies were added to the meta-analysis as a sensitivity assessment $[11,12]$.

\section{Results}

\subsection{Classification of Identified Clinical Studies}

The reviewed trials were categorized by indication, delineating three broad categories (Table 1). Overall, the identified trials investigated the safety and efficacy of the probiotic 
at restoring normal GI function in cases of diarrhea of various etiologies (12 studies; summarized in Table 2) and at supporting immune system function and natural defenses ( 8 studies; summarized in Table 4 ), mainly by enhancing immune competence (6 studies) and reducing the occurrence or recurrence of common infections (2 studies). In addition, other studies (summarized in Table 3) investigated the role of this probiotic combination in infant eczema (one study), oral candidiasis (one study), nutritional iron deficiency anemia (three studies), necrotizing enterocolitis (one study) and hyperbilirubinemia (jaundice; two studies).

\subsection{Studies on Diarrhea of Various Etiologies}

Twelve studies of similar design were conducted in China on children with diarrhea of various etiologies. A total of 1356 infants were enrolled, of whom 714 received a similar probiotic regimen, provided as an adjuvant to a standard of care. These 12 randomized trials (Table 2) assessed efficacy using the same categorical scale, in accordance with the Chinese national standards for the diagnosis and treatment of diarrhea [40], as follows: a "markedly effective" result is characterized by the frequency and characteristics of the stool returning to normal ( $<3$ bowel movements/day) and symptoms disappearing within $72 \mathrm{~h}$ of intervention; an "Effective" result is characterized by a significant improvement in the frequency ( $<4$ bowel movements/day) and characteristics of stool and symptoms within $72 \mathrm{~h}$ of intervention; and an "ineffective" result is characterized by the frequency, characteristics of stools and overall symptoms failing to improve within $72 \mathrm{~h}$ of intervention.

Considering the high level of similarity between the 12 diarrhea studies, we conducted a meta-analysis of the diarrhea treatment outcome (Figure 2a). Overall, probiotic supplementation significantly improved the efficacy of standard diarrhea treatment; pooled results from the 12 studies show a $69 \%$ reduction in the RR of unresolved diarrhea (RR 0.31 [0.23; 0.42$] ; p<0.0001)$, regardless of etiology. Heterogeneity among studies was assessed using $I^{2}$ and $\tau^{2}$ and found absent, which was in accordance with the overall RR and 95\% CI values being the same using either the fixed- or random-effects models.

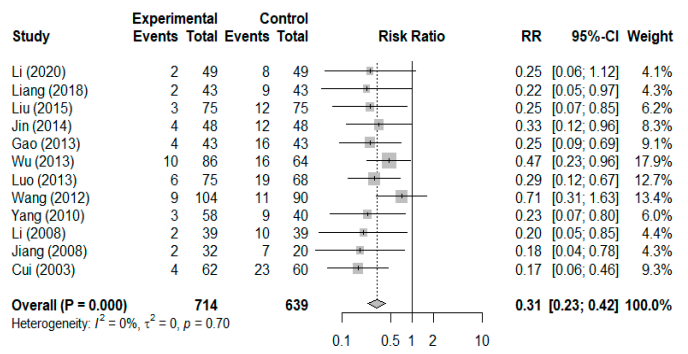

(a)

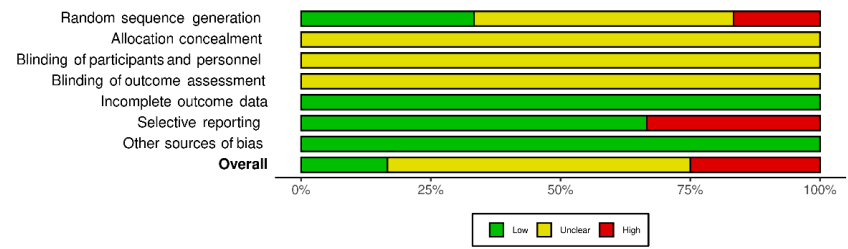

(b)

Figure 2. The probiotic formulation used as adjuvant reduced the relative risk of unresolved diarrhea. (a) Forest plot of studies with an efficacy outcome (effective rate) for diarrhea of various etiologies. Events refers to the number of cases categorized as inefficient (unresolved diarrhea); Total refers to the number of participants in the Experimental (Probiotic) and Control groups. The 12 studies included in the meta-analysis are detailed in Table 2. (References: Cui, 2003 [22], Li, 2008 [24], Jiang, 2008 [26], Yang, 2010 [25], Wang, 2012 [20], Luo, 2013 [19], Gao, 2013 [15], Wu, 2013 [21], Jin, 2014 [23], Liu, 2015 [18], Liang, 2018 [17], Li, 2020 [16]). (b) Mosaic plot showing the risk of bias summary of the studies included in the meta-analysis. 
Table 1. Overview of the clinical studies in pediatric populations.

\begin{tabular}{|c|c|c|c|c|c|}
\hline Indication & Number of Studies & N (Total) & N (Probiotic Arm) & Main Outcome Measures & References \\
\hline \multicolumn{6}{|l|}{ Safety } \\
\hline Healthy children/newborns & 2 & 340 & $\begin{array}{l}\left.\approx 50 / \text { strain }^{[13}\right]^{1} \\
66[14]\end{array}$ & $\begin{array}{l}\text { Growth parameters, adverse events and } \\
\text { serious adverse events, sleep and } \\
\text { crying patterns, D-lactic acid }\end{array}$ & {$[13,14]$} \\
\hline \multicolumn{6}{|l|}{ Gastrointestinal function } \\
\hline Non-infectious diarrhea & 7 & 1001 & 521 & Time to symptom relief, effective rate & [15-21] \\
\hline Rotavirus (RV)-induced diarrhea & 4 & 394 & 207 & Time to symptom relief, effective rate & [22-25] \\
\hline $\begin{array}{l}\text { Persistent diarrhea, } \\
\text { undefined etiology }\end{array}$ & 1 & 52 & 32 & Time to symptom relief, effective rate & {$[26]$} \\
\hline \multicolumn{6}{|c|}{ Immune system function and natural defenses } \\
\hline Secretory $\operatorname{Ig} \mathrm{A}$, cytokines, chemokines & 6 & $405^{2}$ & $224^{2}$ & $\begin{array}{l}\text { Salivary, fecal or serum levels of } \\
\text { secretory IgA, fecal or serum levels of } \\
\text { cytokines/chemokines }\end{array}$ & {$[14,18,27-30]$} \\
\hline Common infections ${ }^{3}$ & 2 & 213 & 140 & $\begin{array}{c}\text { Incidence of infections and related } \\
\text { symptoms, } \\
\text { adverse events }\end{array}$ & {$[31,32]$} \\
\hline \multicolumn{6}{|l|}{ Other indications } \\
\hline Oral candidiasis (thrush) & 1 & 70 & 35 & $\begin{array}{l}\text { Effective rate, recurrence } \\
\text { Eczema Area and Severity Index (EASI) }\end{array}$ & [33] \\
\hline Eczema & 1 & 76 & 38 & $\begin{array}{l}\text { score, } \\
\text { effective rate }\end{array}$ & [34] \\
\hline Necrotizing enterocolitis & 1 & 60 & 30 & $\begin{array}{c}\text { Incidence, severity, mortality, food } \\
\text { tolerance }\end{array}$ & [38] \\
\hline Jaundice & 2 & 1064 & 532 & Incidence, severity (bilirubin levels) & {$[30,39]$} \\
\hline
\end{tabular}

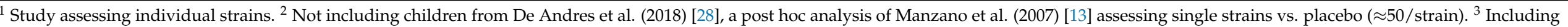
upper respiratory tract infections, ear-nose-throat infections, and gastrointestinal symptoms. 
Table 2. Details of studies on diarrhea of various etiologies.

\begin{tabular}{|c|c|c|c|c|c|c|}
\hline $\begin{array}{c}\text { Reference } \\
\text { [Language] }\end{array}$ & Study Dates & Population & $\begin{array}{l}\text { Study Design } \\
\quad \text { Arms, } n\end{array}$ & Probiotic Regimen & Results (vs. Control) & Adverse Events \\
\hline $\begin{array}{l}\text { Cui, 2003 [22] } \\
\text { [Chinese] }\end{array}$ & $\begin{array}{c}\text { September 2002-November } \\
2002\end{array}$ & $\begin{array}{c}\text { Children } \\
\text { 6-24 months old } \\
\text { RV-ag }^{+} \\
\text {diarrhea } \\
\text { Onset }<72 \mathrm{~h}\end{array}$ & $\begin{array}{c}\text { Randomized, } \\
\text { controlled } \\
\text { Ribavirin + Pro, } n=62 \\
\text { Ribavirin }+ \\
\text { Lacidophilin, } \\
n=60\end{array}$ & $\begin{array}{l}<12 \text { months: } 1 \text { sachet } \mathrm{QD} \\
12-24 \text { months: } 1 \text { sachet } \mathrm{BID} \\
\text { Orally in warm water or milk } \\
\text { Until resolution or up to } 72 \mathrm{~h}\end{array}$ & $\begin{array}{l}\text { Shorter duration of diarrhea }(39.3 \pm \\
17.1 \text { vs. } 63.8 \pm 22.9 \mathrm{~h}) \\
\text { Higher total effective rate }(93.5 \% \text { vs. } \\
\quad 61.7 \% ; p<0.01)\end{array}$ & n.r. \\
\hline $\begin{array}{l}\text { Li, } 2008[24] \\
\text { [Chinese] }\end{array}$ & June 2005-December 2007 & $\begin{array}{l}\text { Children } \\
0-60 \text { months old } \\
\mathrm{RV}_{-} \mathrm{ag}^{+} \\
\text {diarrhea }\end{array}$ & $\begin{array}{c}\text { Randomized, } \\
\text { controlled } \\
\text { Ribavirin + Pro, } \\
n=39 \\
\text { Ribavirin alone, } \\
n=39\end{array}$ & $\begin{array}{l}1 \text { sachet BID } \\
\text { Orally. } 7 \text { days }\end{array}$ & $\begin{array}{l}\text { Higher total effective rate }(94.9 \% \text { vs. } \\
\quad 74.3 \% ; p<0.05)\end{array}$ & n.r. \\
\hline $\begin{array}{l}\text { Jiang, } 2008[26] \\
\text { [Chinese] }\end{array}$ & December 2006-June 2008 & $\begin{array}{c}\text { Children } \\
\text { 3-24 months old } \\
\text { Persistent } \\
\text { diarrhea }\end{array}$ & $\begin{array}{c}\text { Randomized, } \\
\text { active control } \\
\text { Pro, } n=32 \\
\text { GoldenBifido, } n=20\end{array}$ & $\begin{array}{c}<6 \text { months: } 0.5 \text { sachet BID } \\
\text { 6-12 months: } 1 \text { sachet BID } \\
\text { 12-24 months: } 1-2 \text { sachet BID } \\
\text { Until resolution }\end{array}$ & $\begin{array}{c}\text { Shorter duration of diarrhea }(7.14 \pm \\
0.78 \text { vs. } 12.6 \pm 1.75 \mathrm{~d} ; p<0.001) \\
\text { Higher total effective rate }(91 \% \text { vs. } \\
65 \% ; p<0.01)\end{array}$ & n.r. \\
\hline $\begin{array}{l}\text { Yang, 2010 [25] } \\
\text { [Chinese] }\end{array}$ & January 2008-October 2009 & $\begin{array}{l}\text { Children } \\
\text { 6-30 months old } \\
\text { RV-ag+ } \\
\text { diarrhea }\end{array}$ & $\begin{array}{c}\text { Randomized, } \\
\text { controlled } \\
\text { CST + Pro (in formula), } n=58 \\
\text { CST (breastfed or formula), } n= \\
40\end{array}$ & $\begin{array}{c}1 \text { sachet QD } \\
\text { Orally } \\
\text { In lactose-free formula } \\
\text { Duration not stated }\end{array}$ & $\begin{array}{c}\text { Shorter duration of diarrhea }(2.8 \pm 1.1 \\
\text { vs. } 4.9 \pm 2.6 \mathrm{~d} ; p<0.01) \\
\text { Shorter hospital stay }(5.5 \pm 1.7 \text { vs. } 8.5 \\
\pm 2.3 \mathrm{~d} ; p<0.01) . \\
\text { Higher total effective rate }(94.8 \% \text { vs. } \\
77.5 \% ; p \leq 0.05)\end{array}$ & n.r. \\
\hline $\begin{array}{l}\text { Luo, 2013 [19] } \\
\text { [Chinese] }\end{array}$ & April 2010-February 2011 & $\begin{array}{c}\text { Children } \\
\text { 4-36 months old } \\
\text { Non-infectious diarrhea } \\
\text { Onset }<72 \mathrm{~h} \\
\end{array}$ & $\begin{array}{c}\text { Randomized, } \\
\text { controlled } \\
\text { Smecta }^{\circledR}+\text { Pro, } n=75 \\
\text { Smecta }^{\circledR}, n=68\end{array}$ & $\begin{array}{c}<12 \text { months: } 0.33 \text { sachet TID } \\
\text { 12-24 months: } 0.5 \text { sachet BID } \\
24-36 \text { months: } 1 \text { sachet BID } \\
\text { Orally. } 3 \text { days }\end{array}$ & $\begin{array}{l}\text { Higher effective rate } \\
\text { (94.8\% vs. } 73 \% ; p<0.05)\end{array}$ & n.r. \\
\hline $\begin{array}{l}\text { Gao, 2013 [15] } \\
\text { [Chinese] }\end{array}$ & January 2011-January 2012 & $\begin{array}{c}\text { Children } \\
\text { 0-36 months old } \\
\text { Non-infectious diarrhea }\end{array}$ & $\begin{array}{c}\text { Randomized, } \\
\text { controlled } \\
\text { Smecta }^{\circledR}+\text { Pro, } n=43 \\
\text { Smecta }^{\circledR}, n=43\end{array}$ & $\begin{array}{c}<12 \text { months: } 0.33 \text { sachet TID } \\
12-36 \text { months: } 0.5 \text { sachet TID } \\
\text { Orally. } 3 \text { days }\end{array}$ & $\begin{array}{l}\text { Higher total effective rate }(90.7 \% \text { vs. } \\
\qquad 62.8 \% ; p<0.05)\end{array}$ & $\begin{array}{l}\text { None } \\
\text { observed }\end{array}$ \\
\hline $\begin{array}{l}\text { Wu, 2013 [21] } \\
\text { [Chinese] }\end{array}$ & April 2011-December 2011 & $\begin{array}{c}\text { Children } \\
\text { 2-36 months old } \\
\text { Non-infectious diarrhea } \\
\text { Onset }<72 \mathrm{~h}\end{array}$ & $\begin{array}{c}\text { Randomized, } \\
\text { controlled } \\
\text { Smecta }^{\circledR}+\text { Pro, } n=84 \\
\text { Smecta }^{\circledR}, n=64\end{array}$ & $\begin{array}{c}<12 \text { months: } 0.33 \text { sachet TID } \\
12-24 \text { months: } 0.5 \text { sachet BID } \\
24-36 \text { months: } 1 \text { sachet BID } \\
\text { Orally. } 3 \text { days }\end{array}$ & $\begin{array}{l}\text { Higher total effective rate }(90.5 \% \text { vs. } \\
\quad 75 \% ; p<0.05)\end{array}$ & n.r. \\
\hline
\end{tabular}


Table 2. Cont.

\begin{tabular}{|c|c|c|c|c|c|c|}
\hline $\begin{array}{c}\text { Reference } \\
\text { [Language] }\end{array}$ & Study Dates & Population & $\begin{array}{l}\text { Study Design } \\
\quad \text { Arms, } n\end{array}$ & Probiotic Regimen & Results (vs. Control) & Adverse Events \\
\hline $\begin{array}{l}\text { Jin, } 2014 \text { [23] } \\
\text { [Chinese] }\end{array}$ & October 2011-June 2013 & $\begin{array}{c}\text { Children } \\
5-52 \text { months old } \\
\text { (mean } 17.3 \text { mo) } \\
\text { RV-ag } \\
\text { diarrhea. } \\
\text { Onset }<72 \text { h } \\
\end{array}$ & $\begin{array}{c}\text { Randomized, active control } \\
\text { Smecta }^{\circledR}+\text { Ribavirin }+ \text { Pro, } n= \\
48 \\
\text { Smecta }^{\circledR}+\text { Ribavirin, } n=48\end{array}$ & $\begin{array}{l}0.5-1 \text { sachet BID } \\
\text { Orally. In warm water. } \\
\text { Duration not stated. Fluids } \\
\text { provided as needed }\end{array}$ & $\begin{array}{c}\text { Shorter time to symptom relief } \\
\text { (diarrhea, } 31.6 \pm 5.2 \mathrm{~h} \text { vs. } 34.6 \\
\pm 4.1 \mathrm{~h} ; p<0.05) \\
\text { Higher total effective rate } \\
(91.7 \% \text { vs. } 75.0 \% ; p<0.05)\end{array}$ & n.r. \\
\hline $\begin{array}{l}\text { Liu, } 2015 \text { [18] } \\
\text { [Chinese] }\end{array}$ & May 2011-May 2014 & $\begin{array}{c}\text { Children } \\
\text { 3-38 months old } \\
\text { Non-infectious diarrhea }\end{array}$ & $\begin{array}{c}\text { Randomized, } \\
\text { controlled } \\
\text { Smecta }^{\circledR}+\text { Pro, } n=75 \\
\text { Smecta }^{\circledR}, n=75\end{array}$ & $\begin{array}{c}<12 \text { months: } 0.33 \text { sachet TID } \\
12-24 \text { months: } 0.5 \text { sachet BID } \\
24-36 \text { months: } 1 \text { sachet BID } \\
\text { Orally. } 3 \text { days }\end{array}$ & $\begin{array}{c}\text { Higher markedly effective rate } \\
\text { (72\% vs. } 45.33 \% \text {; } p<0<05) \\
\text { Higher total effective rate }(96 \% \\
\text { vs. } 84 \% \text {; } p<0.05) \\
\text { Reduced diarrhea frequency }(p \\
<0.05) \text {, time to diarrhea relief }(p \\
<0.05) \text { and symptom } \\
\text { disappearance }(p<0.05) \text { in the } \\
\text { probiotic group }\end{array}$ & n.r. \\
\hline $\begin{array}{l}\text { Liang, } 2018 \text { [17] } \\
\text { [Chinese] }\end{array}$ & February 2015-May 2017 & $\begin{array}{c}\text { Children } \\
\text { 3-39 months old } \\
\text { Non-infectious } \\
\text { diarrhea }\end{array}$ & $\begin{array}{l}\text { Randomized, active control } \\
\text { Smecta }{ }^{\circledR}+\text { Probiotic, } n=43 \\
\text { Smecta }^{\circledR} \text { alone, } n=43\end{array}$ & $\begin{array}{c}<12 \text { months: } 0.33 \text { sachet TID } \\
\text { 12-24 months: } 0.5 \text { sachet BID } \\
24-36 \text { months: } 1 \text { sachet BID } \\
\text { Orally. } 3 \text { days }\end{array}$ & $\begin{array}{l}\text { Higher total effective rate } \\
(95.35 \% \text { vs. } 79.07 \% ; p<0.05)\end{array}$ & n.r. \\
\hline $\begin{array}{l}\text { Li, } 2020[16] \\
\text { [Chinese] }\end{array}$ & January 2019-February 2020 & $\begin{array}{c}\text { Children } \\
\text { 6-37 months old } \\
\text { Non-infectious diarrhea }\end{array}$ & $\begin{array}{c}\text { Randomized, } \\
\text { controlled } \\
\text { Smecta }^{\circledR}+\text { Pro, } n=49 \\
\text { Smecta }^{\circledR}, n=49\end{array}$ & $\begin{array}{c}<12 \text { months: } 0.33 \text { sachet TID } \\
12-24 \text { months: } \\
0.33 \text { or } 0.66 \text { sachet TID } \\
24-36 \text { months: } 1 \text { sachet TID } \\
\text { Orally. } 3 \text { days }\end{array}$ & $\begin{array}{l}\text { Higher total effective rate } \\
(95.91 \% \text { vs. } 83.67 \% ; p<0.05)\end{array}$ & n.r. \\
\hline
\end{tabular}

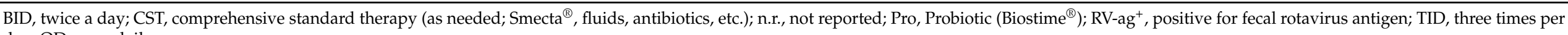
day; QD, once daily. 
Table 3. Details of studies in other indications.

\begin{tabular}{|c|c|c|c|c|c|c|}
\hline $\begin{array}{c}\text { Reference } \\
\text { [Language] }\end{array}$ & Study Dates & Population & $\begin{array}{l}\text { Study Design } \\
\text { Arms }, n\end{array}$ & Probiotic Regimen & Results (vs. Control) & Adverse Events \\
\hline $\begin{array}{l}\mathrm{X}_{\mathrm{i}, 2013[33]}\left[\begin{array}{l}\text { [Chineses] } \\
\text { (Thrush) }\end{array}\right. \\
\text { (Thus }\end{array}$ & January 2011-December 2012 & $\begin{array}{l}\text { Children } \\
1 \text {-26 months old } \\
\text { oral candidiasis infection }\end{array}$ & $\begin{array}{c}\text { Randomized, } \\
\text { controlled } \\
2 \% \mathrm{NaHCO}_{3}+\text { nystatin }+ \text { Pro, } n=35 \\
2 \% \mathrm{NaHCO}_{3}+ \\
\text { nystatin, } n=35 \\
\end{array}$ & $\begin{array}{l}1 \text { sachet BID } \\
\text { Orally. For } 14 \text { days }\end{array}$ & 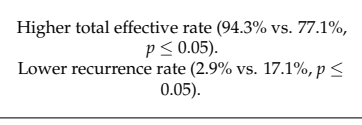 & None observed. \\
\hline $\begin{array}{l}\text { Li, 2017 [34] } \\
\text { [Chineses] } \\
\text { (Eczema) }\end{array}$ & August 2014-January 2016 & $\begin{array}{l}\text { Children } \\
\text { 1-24 month old } \\
\text { infantile eczema }\end{array}$ & $\begin{array}{c}\text { Randomized, } \\
\text { controlled } \\
\text { Topical treatment }+ \text { Probiotic, } n=38 \\
\text { Topical treatment, } n=38\end{array}$ & $\begin{array}{l}1 \text { sachet BID, } \\
\text { for } 2 \text { weeks }\end{array}$ & $\begin{array}{l}\text { Lower EASI scores at } 2 \text { weeks after } \\
\text { treatment } \\
(p<0.05) \\
\text { Higher total effective rate } \\
p<0.01) .\end{array}$ & None observed. \\
\hline $\begin{array}{c}\text { Wu, 2014 [36] } \\
\text { [Chinese] } \\
\text { (IDA) }\end{array}$ & June 2010-April 2013 & $\begin{array}{c}\text { Children } \\
\text { 6-60 months old } \\
\text { Nutritional iron deficiency anemia }\end{array}$ & $\begin{array}{c}\text { Randomized, } \\
\text { controlled } \\
\text { Iron dextran, } n=71 \\
\text { Iron dextran + Probiotic, } n=76\end{array}$ & $\begin{array}{l}1 \text { sachet } \mathrm{QD}, \\
\text { for } 8 \text { weeks }\end{array}$ & $\begin{array}{l}\text { Higher total effective rate }(76.3 \% \text { vs. } 60.6 \% \text {; } \\
\left.\qquad x^{2}=4.236, p=0.040\right)\end{array}$ & $\begin{array}{l}\text { Decreased cases of side effects of Iron } \\
\text { dextran (13.2\% vs. 25.4\%; } p=0.06) \text {. }\end{array}$ \\
\hline $\begin{array}{l}\text { Yang, } 2015[37] \\
\text { [Chinese] } \\
\text { (IDA) }\end{array}$ & February 2011-December 2013 & $\begin{array}{c}\text { Children } \\
6-60 \text { months old } \\
\text { Nutritional iron deficiency anemia }\end{array}$ & $\begin{array}{c}\text { Randomized, } \\
\text { controlled } \\
\text { Iron dextran, } n=76 \\
\text { Iron dextran + Probiotic, } n=76\end{array}$ & $\begin{array}{l}1 \text { sachet BDD, } \\
\text { for } 8 \text { weeks }\end{array}$ & $\begin{array}{l}\text { Higher markedly effective rate }(75 \% \text { vs. } \\
\left.\qquad 55.3 \% ; \chi^{2}=6.453, p=0.011\right)\end{array}$ & $\begin{array}{l}\text { Decreased cases of side effects of iron } \\
\text { dextran } \\
(17.1 \% \text { vs. } 30.3 \% ; p=0.06) \text {. }\end{array}$ \\
\hline $\begin{array}{l}\text { Wei, 2018 [35] } \\
\text { [Chinese] } \\
\text { (IDA) }\end{array}$ & January 2015-December 2016 & $\begin{array}{c}\text { Children } \\
7-72 \text { months old } \\
\text { Nutritional iron deficiency anemia }\end{array}$ & $\begin{array}{c}\text { Randomized, } \\
\text { Controlled } \\
\text { Iron dextran, } n=30 \\
\text { Iron dextran }+ \text { Probiotic, } n=30\end{array}$ & $\begin{array}{c}1 \text { sachet BDD, } \\
\text { for } 8 \text { weeks } \\
\text { All received also Smecta } 9 \text { and antibiotics }\end{array}$ & $\begin{array}{l}\text { Total effective rate } 10 \% \text { higher in probiotic } \\
\text { vs. control }(96.7 \% \text { vs. } 86.7 \% ; p>0.1) \text {. }\end{array}$ & $\begin{array}{l}\text { Decreased cases of side effects of iron } \\
\text { dextran } \\
(20 \% \text { vs. } 40 \% ; p=0.09) \text {. }\end{array}$ \\
\hline $\begin{array}{l}\text { Huang and Ouyang, } 2015 \text { [38] } \\
\text { [Chineses] } \\
\text { (GI function; NEC) }\end{array}$ & August 2011-August 2013 & $\begin{array}{l}\text { Premature newborns (1000-1500 g; mean } 34 \\
\text { weeks gestational age) }\end{array}$ & $\begin{array}{c}\text { Randomized, } \\
\text { placebo-controlled } \\
\text { Placebo, },=30 \\
\text { Probiotic, } n=30\end{array}$ & $\begin{array}{l}0.5 \text { sachet BID, } \\
\text { for } 2 \text { weeks }\end{array}$ & 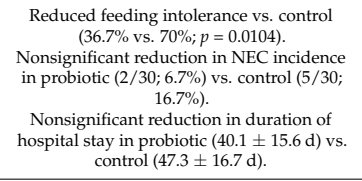 & 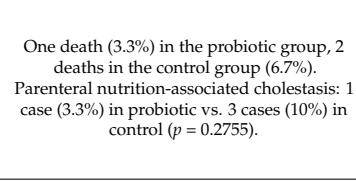 \\
\hline $\begin{array}{l}\text { Gao, 2015 [39] } \\
\text { [Chinese] } \\
\text { (Jaundice) }\end{array}$ & December 2013-December 2013 & $\begin{array}{l}\text { Healthy full-term neonates } \\
\text { gestational age } 37-42 \text { weeks (man } 40 \\
\text { weeks), birth weight } 2500-4000 \mathrm{~g}\end{array}$ & $\begin{array}{l}\text { Randomized, placebo-controlled } \\
\text { Placebo, } n=500 \\
\text { Probiotic, } n=500\end{array}$ & $\begin{array}{l}1 \text { sachet BID, } \\
\text { for } 7 \text { days after birth }\end{array}$ & 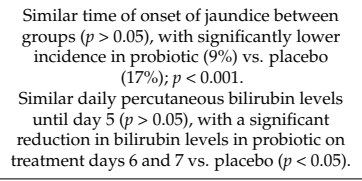 & $\begin{array}{l}\text { Similar between groups (erythema, } \\
\text { vomiting, diarrhha; all } p>0.05 \text { ). }\end{array}$ \\
\hline $\begin{array}{l}\text { Qiu, } 2020[30] \\
\text { [Chinese] } \\
\text { (Jaundice) }\end{array}$ & September 2017-May 2018 & $\begin{array}{l}\text { Healthy full-term neonates } \\
\text { gestational age } 37-41 \text { weeks, aged } \leq 3 \text { days, } \\
\text { with neonatat hyperbilirubinemia } \\
\text { developing within } 24 \mathrm{~h} \text { of birth }\end{array}$ & $\begin{array}{c}\text { Randomized, controlled } \\
\text { Bluu light phototherapy, } n=32 \\
\text { Blue light phototherapy }+ \text { Probiotic, } n=32\end{array}$ & $\begin{array}{l}\begin{array}{l}\text { sachet BID, } \\
\text { for } 5 \text { days }\end{array}\end{array}$ & 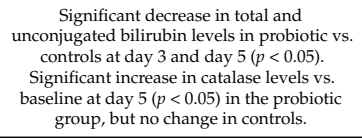 & n.r. \\
\hline
\end{tabular}

BID, twice a day; IDA, iron deficiency anemia; NEC, necrotizing enterocolitis, n.r., not reported; QD, once daily; TID, three times per day. 
Table 4. Details of studies on immunity and natural defenses.

\begin{tabular}{|c|c|c|c|c|c|c|}
\hline $\begin{array}{l}\text { Reference } \\
\text { [Language] }\end{array}$ & Study Dates & Population & $\begin{array}{l}\text { Study Design } \\
\text { Arms, } n\end{array}$ & Probiotic Regimen & Results (vs. Control) & Adverse Events \\
\hline $\begin{array}{l}\text { Chen, } 2007 \text { [27] } \\
\text { [Chinese] }\end{array}$ & Not stated & $\begin{array}{c}\text { Children } \\
0-48 \text { months old } \\
\text { Healthy with low salivary sIgA }\end{array}$ & $\begin{array}{c}\text { Randomized, } \\
\text { controlled } \\
\text { Probiotic, } n=20 \\
\text { No Intervention, } n=8\end{array}$ & $\begin{array}{l}1 \text { sachet BID } \\
\text { Orally. For } 14 \text { days }\end{array}$ & $\begin{array}{l}\text { Increase in salivary sIgA compared to } \\
\text { baseline in probiotic cut tont in controls. } \\
\text { No statistical analyses reported. }\end{array}$ & n.r. \\
\hline Cazzola, 2010 [31] [English] & December 2006-March 2007 & $\begin{array}{c}\text { Children } \\
3-7 \text { years old } \\
\geq 3 \text { infections (ENII, URII, GI illness) in } \\
\text { past winter. }\end{array}$ & $\begin{array}{l}\text { Randomized, double-blind, } \\
\text { Placebo-controlled } \\
\text { Probiotici, } n=62 \\
\text { Placebo, } n=73\end{array}$ & $\begin{array}{l}1 \text { sachet QD } \\
\text { Orally. For } 3 \text { months }\end{array}$ & 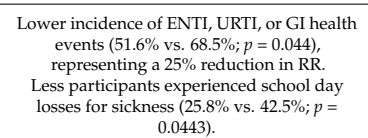 & $\begin{array}{l}\text { 2SAEs; } 1 \text { abdominal pain in placebo and } 1 \\
\text { otitis media in Probiotic. } \\
24 \mathrm{AEs} \text { in } 20 \text { children }(9 \text { in placebo, } 11 \text { in } \\
\text { Probiotic), most were expected respiratory } \\
\text { or GI events. }\end{array}$ \\
\hline Pantovic, 2013 [29] [English] & Not stated & $\begin{array}{c}\text { Children } \\
6-42 \text { months old } \\
\text { low IgA levels } \\
\text { hospitalized for URTI or ENTI }\end{array}$ & $\begin{array}{l}\text { Open-label, uncontrolled before-after study } \\
\text { Probiotic, } n=31\end{array}$ & $\begin{array}{l}1 \text { sachet QD } \\
\text { Orally. For } 6 \text { months }\end{array}$ & $\begin{array}{l}\text { Increase in serum IgA levels in } 35 \% \text { of the } \\
\text { children after } 3 \text { months and } 18 \% \text { after } 6 \\
\text { months }(p<0.05) \text {, normalized to normal } \\
\text { range. } \\
\text { Clinical immprovement in URTI after } 3 \\
\text { months, and no infections diagnosed } \\
\text { between } 3 \text { and } 6 \text { months. }\end{array}$ & n.r. \\
\hline $\begin{array}{l}\text { Liu, } 2015[18] \\
\text { [Chinese] }\end{array}$ & May 2011-May 2014 & $\begin{array}{l}\text { Children } \\
\text { 3-38 months old } \\
\text { Non-infectious } \\
\text { diarrhea }\end{array}$ & 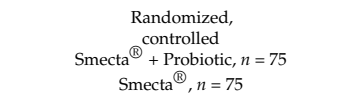 & $\begin{array}{l}<12 \text { months: } 0.33 \text { sachet TII } \\
13-24 \text { months: } 0.5 \text { sachet BID } \\
24-36 \text { month: sachet BID } \\
\text { Orally. For } 3 \text { days }\end{array}$ & $\begin{array}{l}\text { Lower serum levels of pro-inflammatory } \\
\text { cytokines sL-6 and IL-17 } \\
\quad p<0.05) \\
\text { Higher levels of salivary sIgA }(p<0.05) \text {. }\end{array}$ & n.r. \\
\hline Stojkovic, 2016 [32] [English] & Not stated. & $\begin{array}{c}\text { Children } \\
<5 \text { years } \\
\text { Hospitalized during the past year for } \\
\text { respiratory diseases }\end{array}$ & $\begin{array}{c}\text { Open label, } \\
\text { before-after } \\
\text { Probiotic, } n=78 \\
\text { Divided into } 3 \text { groups based on medical } \\
\text { history } \\
\text { G1: URRTI +wherezing, } n=50 \\
\text { G2: URTI w/o wheezing, } n=17 \\
\text { G3: Wheezing w/o URTI, } n=11\end{array}$ & $\begin{array}{l}1 \text { sachet } \mathrm{QD} \\
\text { Orally. For } 9 \text { months }\end{array}$ & 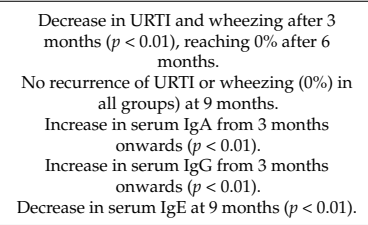 & None observed. \\
\hline $\begin{array}{l}\text { Manzano, 2017 [13]; } \\
\text { De Andres, 2018 [28] } \\
\text { [English] }\end{array}$ & August 2014-December 2016 & $\begin{array}{l}\text { Children } \\
\text { 3-12 months old } \\
\text { Healthy }\end{array}$ & $\begin{array}{c}\text { Randomized, double-blind, } \\
\text { placebo-controlled } \\
\text { L. helveticus Rosell } \\
\text { B. infantis Rosel| }{ }^{\Theta}-52, n=52 \\
\text { B. bifidum Rosell }{ }^{\circledR}-71, n=53 \\
\text { Placebo, } n=52\end{array}$ & $\begin{array}{l}3 \times 10^{9} \text { CFU of each single strain QD } \\
\text { Orally. For } 8 \text { weeks }\end{array}$ & 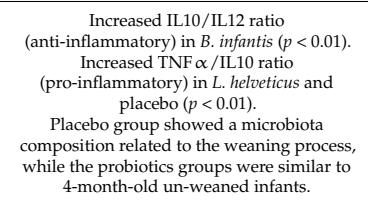 & $\begin{array}{l}\text { No difference between groups for the } \\
\text { number and severitit of adverse tevents } \\
\text { (mild) nor in behavioral and anthropometric } \\
\text { parameters. No SAEs observed. }\end{array}$ \\
\hline $\begin{array}{l}\text { Xiao, 2019 [14] } \\
\text { [English] }\end{array}$ & December 2014-November 2015 & $\begin{array}{l}\text { Children } 3.5-6 \text { months old } \\
\text { Healthy }\end{array}$ & $\begin{array}{c}\text { Randomized, placebo-controlled } \\
\text { Probiotic, } n=66 \\
\text { Placebo, } n=66\end{array}$ & $\begin{array}{l}1 \text { sachet } \mathrm{QD} \\
\text { Orally, in formula. } \\
\text { For } 4 \text { weeks }\end{array}$ & $\begin{array}{l}\text { Maintained higher fecal IIIA levels at the } \\
\text { end of the four-week treatment period }(p< \\
0.05) \text {. }\end{array}$ & $\begin{array}{c}\text { All AEs reported were minor and more } \\
\text { frequent in the placebo group. } \\
\text { Probiotic: } 37 \mathrm{AEs} ; 2 \text { respiritary, } 12 \mathrm{GI}, 4 \\
\text { dermatological. } \\
\text { Placebo: } 69 \mathrm{AEs}, 38 \text { respiratory, } 15 \mathrm{GI}, 16 \\
\text { dermatological. } \\
\text { No effect on growth rate. }\end{array}$ \\
\hline $\begin{array}{l}\text { Qiu, } 2020[30] \\
\text { [Chinese] }\end{array}$ & September 2017-May 2018 & Full-term neonates with hyperbilirubinemia & $\begin{array}{l}\text { Randomized, } \\
\text { controlled } \\
\text { Blue light photo- } \\
\text { therapy }+ \text { Probioitic, } n=32 \\
\text { Blue light photo- } \\
\text { therapy, } n=32\end{array}$ & $\begin{array}{l}1 \text { sachet BID, } \\
\text { for } 5 \text { days }\end{array}$ & 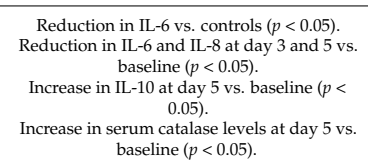 & n.r. \\
\hline
\end{tabular}

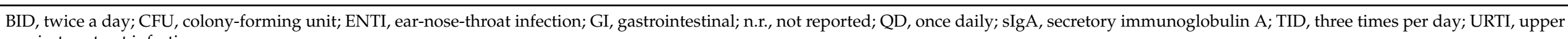
respiratory tract infection. 
For the risk of bias analysis (Figure 2b), most studies included in the meta-analysis were categorized as low or unclear risk [15-18,20,22-24,26], with three studies classified as high risk of bias due to potential randomization or selective reporting issues [19,21,25]. All studies were classified as unclear risk for the allocation concealment and blindingrelated domains; a similar trend was observed in a previous systematic review, which could reflect regional publication practices [41]. Publication bias was assessed using the funnel plot method (Figure 3) and asymmetry was found significant (Egger's regression test; $\mathrm{t}=-2.84, \mathrm{df}=10, p=0.0176)$, suggesting the potential occurrence of publication bias. The number of missing studies to correct the asymmetry was estimated to five using the Trim and Fill method by Duval and Tweedie [11,12]. Upon inclusion of 5 negative studies in the meta-analysis (empty circles; total studies $=17$ ), the result remained significant with the reduction in the risk of unresolved diarrhea changing from $69 \%$ to $62.5 \%$ (RR 0.3753 [0.2867; 0.4913]; $p<0.0001)$.

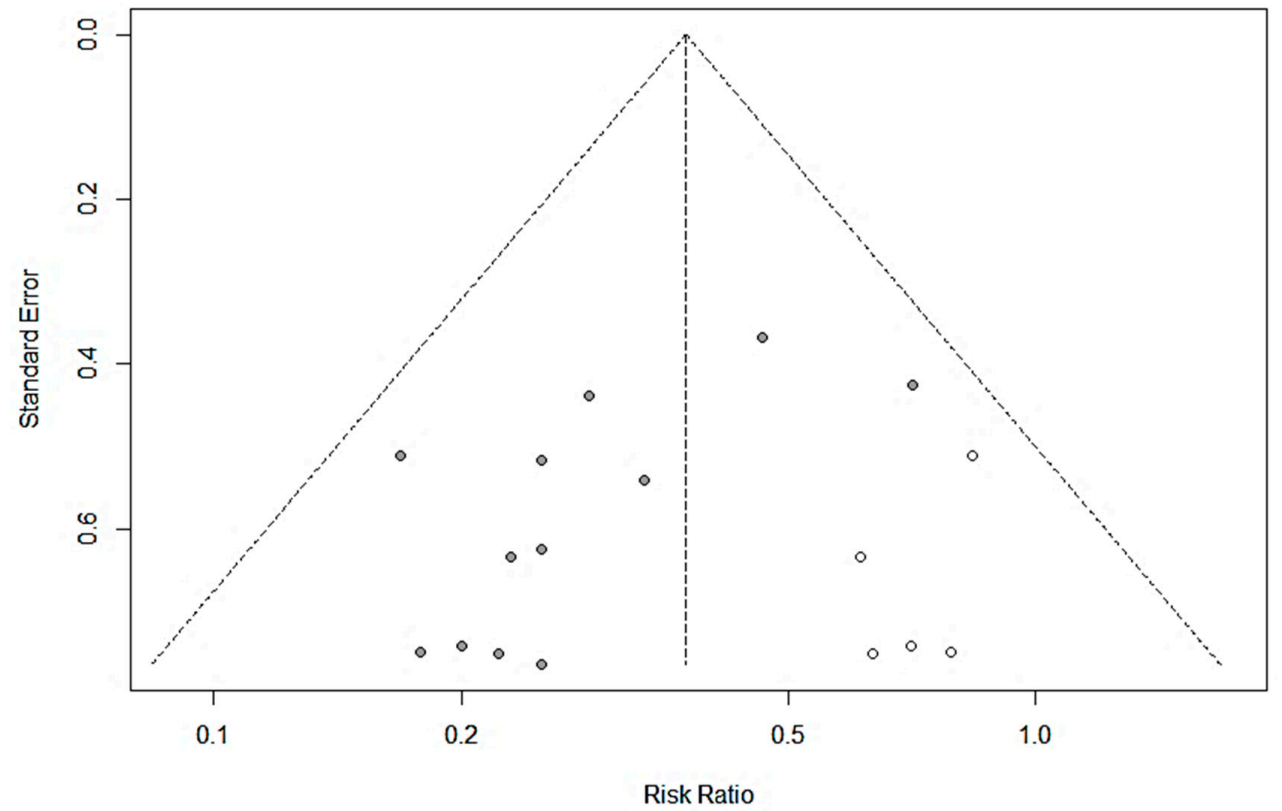

Figure 3. Funnel plot showing the relationship between the relative risk and its standard error for the 12 studies included (filled circles) and the potential 5 negative studies added to correct asymmetry using the Trim and Fill method (empty circles).

\subsection{Studies on Immune Competence and Inflammatory Markers}

All studies classified in the immunity and natural defenses indications are detailed in Table 4. At the mucosal interface, secretory immunoglobulin A (sIgA) mediate humoral immunity by regulating the balance between immune exclusion of pathogenic microorganisms and tolerance to commensal bacteria, thereby favoring the establishment of a symbiotic relationship between the gut microbiota and the host [42,43]. At birth, immunoglobulins (Ig), and mainly secretory $\operatorname{IgA}(\operatorname{sg} \mathrm{A})$, are provided by the mother's breast milk while the infant's own production of intestinal sIgA by the gut-associated lymphoid tissue (GALT) develops [44,45]. In formula-fed infants, the immune protection provided by the mother is absent and the development of infant formula able to promote microbial colonization by Bifidobacteria and stimulate the development of immune competence is required. Three studies $[14,18,27]$ assessed the effect of the probiotic on the development of mucosal immune competence using sIgA levels in the saliva or feces as a surrogate endpoint (Table 4). Among these studies, two were conducted in healthy infants (0-48 months old) [27] or healthy formula-fed babies (3-6 months old) [14], and one in children with non-infectious diarrhea (3-38 months old) [18].

Overall, supplementation with the probiotic increased or maintained higher sIgA levels, suggesting an enhanced mucosal immunity. Chen et al. (2007), in a pilot-like study, 
reported a significant increase in salivary sIgA levels in children of all age groups compared to randomly selected age-matched controls [27]. The study conducted by Xiao et al. (2019) enrolling 132 formula-fed infants showed that formula-fed infants receiving the probiotic maintained significantly higher fecal sIgA levels at the end of the 4-week supplementation period compared to those receiving placebo, suggesting a positive effect of probiotics on intestinal sIgA production [14]. Similarly, Liu et al. (2015) reported a significant increase in salivary sIgA after 3 days of supplementation in children with non-infectious diarrhea [18]. They also assessed the levels of inflammatory markers; the pro-inflammatory cytokines IL-6 and IL-17 were significantly reduced in the probiotic group at the end of treatment compared to controls [18].

The modulation of inflammatory marker was also assessed in a post hoc analysis of a placebo-controlled safety study cohort of healthy 3-12-month-old children receiving the individual strains for 8 weeks [13,28]. De Andres et al. (2018) reported that the IL10/IL-12 anti-inflammatory ratio increased significantly with $B$. infantis Rosell ${ }^{\circledR}-33$ but decreased with placebo [28]. Interestingly, the authors also reported that the microbiota composition in children receiving the probiotic strains was similar to the microbiota profile typical of a 4-month-old, un-weaned infant, while the profile in the placebo group corresponded to that seen during the weaning process with a decrease in different Bifidobacterium species, such as $B$. bifidum and $B$. breve, and an increase in genera seen in the more diversified microbiota of adults (Bacteroides, Blautia, Clostridium, Coprococcus and Faecalibacterium) [28].

Regulation of immune function was also assessed using circulating levels of IgA in the serum. Although less is known about the role of serum IgA in regulating the systemic inflammatory response, low levels of serum IgA compared to other serum immunoglobulins have been observed in children with selective IgA deficiency, who are known to be more susceptible to sinopulmonary infections, GI illnesses, and allergic diseases [46,47]. Two studies conducted in Serbia [29,32] assessed the effect of this formulation on serum immunoglobulin levels in infection-prone children with low serum IgA levels. In both studies, most of the enrolled children were classified as atopic based on a positive sensitization response to 25-30 allergens in a skin prick test and/or elevated IgE levels. Pantovic et al. (2013) reported a normalization of serum IgA levels in 35\% of the children after 3 months and $81 \%$ after 6 months [29]. Stoijkovic et al. (2016) reported a statistically significant decrease in $\operatorname{IgE}$ after 9 months of supplementation, and a rise in serum $\operatorname{IgA}, \operatorname{IgG}$, and $\operatorname{IgM}$ levels after 3 months of supplementation, which was maintained over the following 6 months [32]. While Pantovic et al. (2013) did not correlate the effects of the probiotic on serum IgA levels with the incidence of infections, two studies, including Stojkovic et al. (2016), have shown the efficacy of this probiotic against respiratory infections in frequently sick children.

\subsection{Studies on the Prevention of Common Infections}

Cazzola et al. 2010 [31] and Stojkovic et al. 2016 [32] investigated the efficacy of the probiotic (administered from 3 to 9 months) at preventing common respiratory infections or complications in children, such as upper respiratory tract infections (URTI), ear/nose/throat infections (ENTI), and GI illnesses. In France, Cazzola et al. (2010) enrolled 135 children diagnosed with $>3$ infections in the past winter to receive either probiotic $(n=62)$ or placebo $(n=73)$ for 3 months. There was a significant reduction of $24.7 \%$ $(p \leq 0.045)$ in the relative risk (RR) of occurrence of common infectious diseases in the probiotic group, with $51.6 \%(32 / 62)$ of children reporting at least one infection versus $68.5 \%(50 / 73)$ in the placebo group. Furthermore, $25.8 \%$ in the probiotic group $(p \leq 0.043)$ reported missing at least one school day for sickness versus $42.5 \%$ in placebo, representing a $40 \%$ decrease in school absenteeism [31]. Stojkovic et al. (2016) supplemented probiotics for 9 months in 78 hospitalized children aged 1.5 months to 5 years. All children were categorized into three groups according to the predominance of symptoms over the past year; Group I-predominance of respiratory infection and wheezing; Group IIpredominance of respiratory infection without wheezing; Group III-predominance of 
wheezing without accompanying respiratory infection. The elevation in serum IgA levels in children from Groups I and II described above was correlated with a decline of clinical symptoms' occurrence. In children from Group III a significant rise in serum IgA levels was observed only within 6-9 months and was correlated with a statistically significant decrease in the frequency of wheezing episodes at the end of the study [32]. This openlabel before-after study shows that supplementation for 3-9 months results in a positive impact on the inflammatory status in children that is consistent with the improvement in clinical symptoms.

\subsection{Studies in Other Indications}

The studies classified in the category "other indications" are described below and summarized in Table 3.

\subsubsection{Oral Candidiasis (Thrush)}

A Chinese study tested the probiotic for oral candidiasis infections (oral thrush) in 70 children (1-26 months old) [33]. The children were randomized into two groups of 35 receiving $2 \%$ sodium bicarbonate with nystatin (standard of care) with or without probiotic. Both the effective rate $(94.3 \%$ vs. $77.1 \%, p<0.05)$ and the recurrence rate $(2.9 \%$ vs. $17.1 \%, p<0.05)$ significantly favored the probiotic arm. The authors concluded that the probiotic was effective as adjuvant to nystatin and sodium bicarbonate in the treatment and prevention of oral thrush in children.

\subsubsection{Eczema}

$\mathrm{Li}$ (2017) conducted a randomized controlled study to assess the effect of the probiotic ( 2 weeks) as an adjuvant to a standard topical treatment in 76 children with eczema (38/group) [34]. Baseline characteristics were similar between groups $(p>0.05)$. The Eczema Area and Severity Index (EASI) scores were significantly improved in the probiotic group compared to controls at 1 and 2 weeks after treatment $(p<0.05)$. The total effective rate in the intervention group was $92.1 \%(35 / 38)$ compared to $65.7 \%(25 / 38)$ in controls $(p<0.01)$.

\subsubsection{Iron Deficiency Anemia}

Three studies of similar design investigated the effect of the probiotic as adjuvant to iron dextran supplementation for 8 weeks in a total of 182 children (6-60 months old) hospitalized for nutritional iron deficiency anemia (IDA) [35-37]. In parallel, children randomized to the control arms $(n=182)$ received iron dextran supplementation (standard of care) without probiotic. In all studies, the average age of enrolled children was approximately 3 years old, and the baseline characteristics were found similar between study arms in terms of age, gender distribution and severity of anemia $(p>0.05)$. Similar parameters to determine the efficacy of supplementation (i.e., blood anemia parameters) were used, including blood hemoglobin levels, mean corpuscular volume, mean corpuscular hemoglobin concentrations, reticulocyte counts, and serum iron levels. Treatment efficacy was measured as rates using a categorical scale, with a "markedly effective" result characterized by all symptoms and blood indicators returning to normal levels, an "effective" result characterized by an improvement in symptoms accompanied by a rise in hemoglobin levels by more than $20 \mathrm{~g} / \mathrm{L}$ above baseline levels, and an "ineffective" result, by the absence of improvement in symptoms or by hemoglobin levels not increasing by more than $20 \mathrm{~g} / \mathrm{L}$ above baseline levels [35-37].

To compare these studies, ineffective cases were used to calculate the RR of treatment failure in the absence of probiotic (Figure 4a). When combining all three studies to increase the number of participants, the probiotic significantly reduced the risk of unresolved anemia by $49 \%$ (RR 0.51 [0.28-0.92]; $p=0.0263$ ). Considering the high efficiency of iron dextran supplementation on its own, improvement of treatment efficacy with the probiotic (i.e., promoting iron absorption) is considered clinically meaningful. Furthermore, the common adverse effects of iron dextran supplementation, namely GI symptoms (diarrhea or constipation), nausea, 
and metal taste, were monitored in all 3 studies and found consistently reduced with the probiotic (Figure 4b), with a 47\% reduction in the risk of side effects (RR 0.53 [0.37-0.77]; $p=0.0009$ ) [35-37]. All three studies were found at low risk of bias (Figure 4c), except for the domains related to blinding which are typically not reported possibly due to regional reporting practices, as observed previously [41].

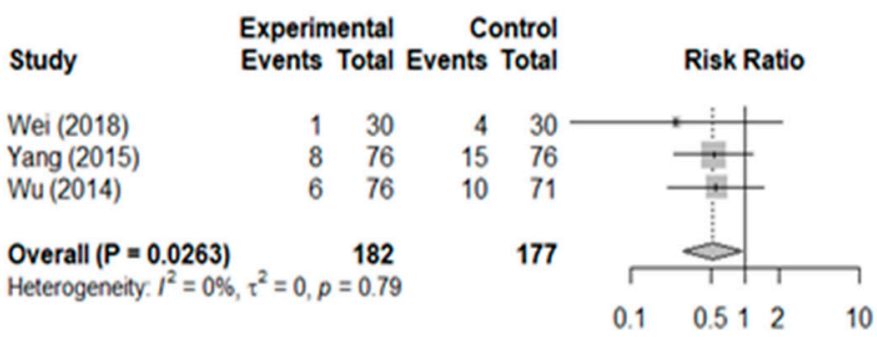

(a)

\begin{tabular}{lrc} 
Study & \multicolumn{2}{c}{$\begin{array}{c}\text { Experimental } \\
\text { Events }\end{array}$} \\
Wei $(2018)$ & 6 & 30 \\
Yang $(2015)$ & 13 & 76 \\
Wu $(2014)$ & 14 & 76 \\
& & \\
Overall $(P=\mathbf{P}=0.000)$ & \multicolumn{1}{c}{$\mathbf{1 8 2}$} \\
Heterogeneity: $I^{2}=0 \%, \tau^{2}=0, p=0.97$
\end{tabular}

\section{Control}

ents Total
Risk Ratio

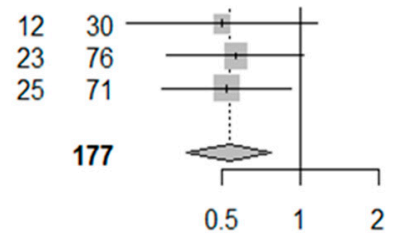

\section{RR $\quad 95 \% \cdot \mathrm{Cl}$ Weight}

$0.25[0.03: 2.11] \quad 7.6 \%$

$0.53[0.24 ; 1.18] \quad 54.6 \%$

$0.56[0.21,1.46] \quad 37.7 \%$

$0.51[0.28 ; 0.92] 100.0 \%$
RR $\quad 95 \%-C l$ Weight

$0.50[0.22 ; 1.16] \quad 19.5 \%$

$0.57[0.31 ; 1.03] \quad 38.0 \%$

$0.52[0.30 ; 0.92] \quad 42.5 \%$

$0.53[0.37 ; 0.77] 100.0 \%$

(b)

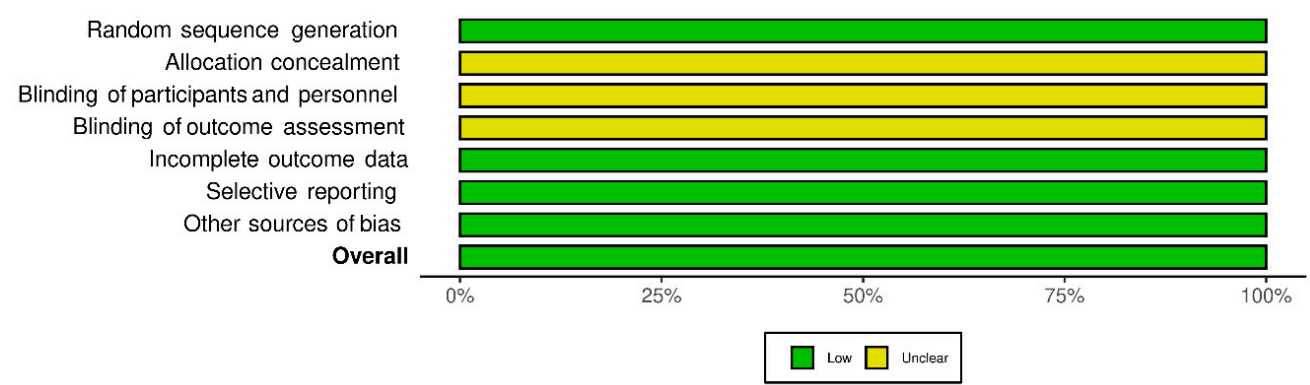

(c)

Figure 4. The probiotic reduced the relative risk of unresolved anemia and side effects in children with nutritional iron deficiency anemia receiving iron supplementation. Forest plot of studies on IDA treatment efficacy and occurrence of side effects. The 3 studies included in these meta-analyses are detailed in Table 3. (References: Wei, 2018 [35], Yang, 2015 [37], Wu, 2014 [36]). (a) Events refers to the number of cases categorized as inefficient (negative outcome of treatment); Total refers to the number of participants in the Experimental (Probiotic) and Control groups. (b) Events refers to the number of cases experiencing iron supplementation-related side effects; Total refers to the number of participants in the Experimental (Probiotic) and Control groups. (c) Mosaic plot showing the summary of the risk of bias analysis of studies included in the meta-analysis.

\subsubsection{GI Function and Necrotizing Enterocolitis in Newborns}

In a randomized placebo-controlled trial, Huang et al. (2015) evaluated the safety and efficacy of the probiotic during 2 weeks on the GI function of 60 very low birth weight (VLBW) newborns ( $n=30 / \mathrm{arm} ; 1000-1500 \mathrm{~g}$; average 34 weeks gestational age) [38]. All participants received intravenous nutrition starting on the second day after birth. Although not found statistically different, the incidence of necrotizing enterocolitis (NEC) was lower in the probiotic versus controls $(6.7 \%$ vs. $16.7 \% ; p>0.05)$ with a relatively shorter hospital stay duration compared to controls ( $40.1 \pm 15.6$ vs. $47.3 \pm 16.7$ days; $p>0.05$ ). 
Adverse events were similar between groups, with one death (3.3\%) in the probiotic group and two $(6.7 \%)$ in the controls. There was a slightly lower incidence of parenteral nutritionassociated cholestasis (PNAC) in the probiotic group with only 1 case (3.3\%) vs. 3 cases $(10 \%)$ in controls $(p>0.05)$. Feeding intolerance, which was assessed by the occurrence of GI symptoms (i.e., vomiting, diarrhea, or constipation), was significantly lower in the probiotic vs. controls ( $36.7 \%$ vs. $70 \% ; p=0.0104)$. Results suggest a positive effect of this formulation on the GI function in VLBW infants, which supports the conduct of further clinical trials assessing the effect of this probiotic formulation on the incidence and severity of NEC and PNAC in larger cohorts [38].

\subsubsection{Jaundice in Newborns}

In a double-blind, placebo-controlled trial, Gao et al. (2015) assessed the safety and efficacy of a 7 day prophylaxis with the probiotic for the prevention of jaundice in a population of 1000 healthy full-term neonates ( $n=500$ /group; $2500-4000$ g; 37-42 weeks gestational age) [39]. The time of onset of jaundice was similar between groups $(p>0.05)$, but incidence was significantly lower $(p<0.001)$ in probiotics $(9 \%)$ versus controls $(17 \%)$. The average levels of daily percutaneous bilirubin from the second to fifth postnatal day were similar in both groups $(p>0.05)$ but the average levels of daily percutaneous bilirubin on the sixth and seventh day after birth were significantly lower in probiotics versus controls $(p<0.05)$. Adverse events were similar between groups $(p>0.05)$. This probiotic formulation was well tolerated, clinically safe, and reduced the daily percutaneous bilirubin levels and incidence of hyperbilirubinemia in newborns [39].

Qiu et al. (2020) conducted a study on the efficacy of the probiotic as an adjuvant to blue light phototherapy $\left(450-475 \mathrm{~nm}\right.$; light intensity $\left.10-12 \mu \mathrm{W} / \mathrm{cm}^{2} / \mathrm{nm}\right)$ in 64 fullterm otherwise healthy newborns (gestational age 37-41 weeks) diagnosed with jaundice ( $n=32 / \mathrm{arm})[30,48]$. Briefly, only children younger than 3 days old, in whom appearance of jaundice occurred within $24 \mathrm{~h}$ from birth, with a total bilirubin level above $102 \mu \mathrm{mol} / \mathrm{L}$, a daily rise in bilirubin above $85 \mu \mathrm{mol} / \mathrm{L}$, and conjugated bilirubin levels above $25 \mu \mathrm{mol} / \mathrm{L}$ were included. Exclusion criteria were congenital biliary malformations, acute bilirubin encephalopathy with central nervous system (CNS) manifestations, a score lower than 7 on the Agpar test (one minute), cytomegalovirus infections, or severe infections (e.g., septic shock, CNS infections). Although jaundice improved in both groups, there was a significantly higher reduction in the total and conjugated bilirubin levels at 3 and 5 days of treatment in the probiotic group $(p<0.05)$. There was also a significant reduction in IL-6 levels in the probiotic group compared to controls $(p<0.05)$ and a significant reduction in IL-6 and IL-8 levels at day 3 and 5, and a significant increase in IL-10 at day 5 vs. baseline in the probiotic group (all $p<0.05$ ), but not in the controls. Oxidative stress markers were unchanged in both groups, except for serum catalase levels at day 5 , which were significantly higher in the probiotic group compared to baseline $(p<0.05)$ [30]. Overall, the probiotic formulation was found safe and effective as an adjunct to blue light phototherapy for jaundice in otherwise healthy full-term newborns, possibly by improving the inflammatory status or, more speculatively, oxidative stress levels.

\section{Discussion and Concluding Remarks}

The clinical studies reviewed herein demonstrate the efficacy of this formulation as an adjuvant to standard treatment for diarrhea of various etiologies [15-26] and support its role at sustaining natural defenses against infections and boosting immunity $[13-15,17-19,21-26,28$ 32]. Promising results support additional studies on this probiotic formulation in children with oral candidiasis [33], iron deficiency anemia [35-37], eczema [34], as well as for preventing jaundice or improving GI function in newborns [30,38,39].

The safety of this formulation has been confirmed in several studies and through the pharmacovigilance program in place [7]. While adverse events monitoring is expected to have been conducted in all trials, some studies have not adequately reported on this aspect. However, in the 13 studies where adverse events monitoring was reported [13-15,20,31-39] few adverse 
events were observed, and none could be attributed to the intervention over placebo. While the systematic reporting of adverse events in clinical trials generally needs to be improved, there is no evidence of increased risk resulting from probiotic use in otherwise healthy children [49]. However, caution is generally recommended for all probiotics in individuals more susceptible to bacterial infections, such as children younger than 3 years old with short bowel syndrome or in severely immunocompromised individuals and immunosuppressed patients [50,51]. The innocuity of the strains in this formulation is recognized by several authoritative bodies worldwide, where the strains are included in lists of safe strains for consumption in foods. Probiokid $^{\circledR}$ and the individual strains have been recognized for their safety by the US FDA in the form of a no question letter for notified GRAS status for use in non-exempt infant formula, and in Canada from the NNHPD for use in Natural Health Products in infants 3 months old and over.

This review has strengths and limitations. A strength of this review is the cohesion between studies allowing to conduct outcome-specific meta-analyses without heterogeneity. Indeed, several meta-analyses attempt to compare studies using several different probiotics and measuring clinical outcomes with a variety of methods. Here, the studies are homogeneous in terms of probiotic intervention, study designs and outcome assessment methods, which strengthens the conclusions reached by individual studies, most notably with regard to the benefits on diarrhea treatment. Except for 2 studies that did not provide details on the diagnosis criteria used to define acute diarrhea, the 10 remaining studies used local guidelines in place for the diagnosis of diarrheal diseases. Another limitation of this review is the fact that many studies were assigned an unclear of high risk of bias. In many studies, while randomization was used, reporting was deficient in terms of the description of the randomization method, allocation concealment or blinding procedures implemented. This could potentially be explained by the existence of country-specific reporting guidelines. Nevertheless, the flow of participants throughout the studies and clinical outcomes were well described, which suggests a generally low risk of attrition and reporting biases in outcomes.

For the meta-analysis of IDA studies, the risk of bias was found low in all domains, heterogeneity was absent, and the results were identical when fixed- or random-effects models were applied. However, it is known that standard random-effects meta-analyses with few studies ( $n=2$ or 3 ) are less accurate, especially in the presence of heterogeneity or when studies are highly variable in size [52]. Hence, while the current positive effects in children with IDA receiving iron supplementation are promising, the conclusion about the efficacy of the formulation in this specific indication could change with an increased number of studies.

This probiotic formulation was mostly studied in children. However, a similar effect of the strain B. bifidum Rosell $^{\circledR}-71$ was shown in healthy young adults undergoing university exams; this strain significantly increased the proportion of healthy days (i.e., without cold and flu symptoms) and reduced diarrhea symptoms associated with stress [53,54]. Mechanistic insights on the modes of action of this probiotic formulation were obtained from in vitro and in vivo studies. Briefly, the formulation and individual strains were shown to preserve the integrity of the intestinal barrier, and to exert immunomodulatory functions in vitro and in vivo, which may also contribute to the beneficial effects observed on diarrhea symptoms and overall GI and immune health. In vitro, this probiotic was shown to counteract Toll-like receptor 3 (TLR3)-induced inflammation triggered by the doublestranded RNA ligand Poly(I:C), which mimics a Th1 and antiviral innate immune response in human intestinal epithelial cells $[55,56]$. In vivo, the formulation induced mucin and tight junction protein expression, and suppressed the inflammation and apoptosis induced by the carcinogenic compound dimethylhydrazine dihydrochloride [57]. Furthermore, this probiotic increased anti-inflammatory cytokines (i.e., IL-4) and reduced pro-inflammatory cytokines (e.g., IL-1a, IL-1b, IL-6, IFN- $\gamma$ and TNF- $\alpha$ ) in rats infected with enterotoxic E. coli (model of a Th1 immune response), and reduces pro-inflammatory cytokines (e.g., IL-1a) in rats infected with the pathogen $N$. brasiliensis (model of a Th2 immune response) [58]. 
In addition to regulating host defenses, probiotics' effects on the immune systems also influence the host in a systemic manner. Recently, an in vivo study on this probiotic formulation revealed that the immunomodulatory effects of this formulation could also underly a potential role in indications related to the microbiota-gut-brain axis (MGBA). Indeed, this probiotic formulation prevented the increase in pro-inflammatory cytokines in the dam's serum and fetal brain in the maternal immune activation (MIA) mouse model of autism [59]. In addition, it prevented the neuronal loss and the reduction in GABA levels seen in the MIA adult offspring's prefrontal cortex. These changes were correlated with behavioral improvements compatible with an amelioration in autism-like symptoms in these mice (social deficits and stereotypical behavior), but also reduced anxiety- and depression-related behaviors in the adult MIA offspring. This study, by linking the immunomodulatory and behavioral effects of Probiokid ${ }^{\circledR}$ with the microbiotagut-brain axis in a pre-clinical setting, provides a rationale to explore the potential role of this formulation in other MGBA models. Of note, the limited number of clinical trials conducted so far in children with ASD have resulted in conflicting results about the potential of probiotics to improve GI or behavioral symptoms in this population [60]. In addition, the beneficial effects on GI function with a trend towards a reduction in NEC incidence in a small cohort of premature infants [38], along with the reduction in the severity of intestinal tissue damage in a rodent model of NEC [61] warrant further studies on this probiotic in premature infants at risk of NEC.

Overall, the current knowledge base available on this probiotic indicates its efficacy to produce clinical benefits in various indications frequently seen in children, including diarrhea of various etiology and respiratory or other infections. The promising positive effects seen in a variety of conditions commonly affecting newborns and children warrants more studies on this probiotic to better understand its mechanisms of action in immune modulation and gastrointestinal protection, but also at the systemic level based on preclinical data. However, there is currently a gap in knowledge about how microbiotaderived molecules may systemically affect the host, especially considering the complexity of these interactions and variable translatability of mechanisms from animal models to humans. The recent advents in "meta-omics" technologies and use of various experimental models [62-65] could help bridge this gap.

Supplementary Materials: The following are available online at https://www.mdpi.com/article/10 .3390/nu13061908/s1, Table S1: PubMed search strategy.

Author Contributions: Conceptualization, A.T. and T.A.T.; methodology/literature searches, A.T. and X.X.; data curation, A.T. and X.X.; data analysis, A.T., X.X. and J.C.; writing-original draft preparation, A.T.; writing - review and editing, A.T., X.X., J.C. and T.A.T.; supervision, T.A.T. All authors have read and agreed to the published version of the manuscript.

Funding: This review received no external funding.

Conflicts of Interest: A.T., X.X., and T.A.T. are employed by Lallemand Health Solutions Inc., a company that researches, develops, manufactures, markets, and sells probiotics (including Probiokid ${ }^{\circledR}$ and its individual strains) to business clients but not directly to consumers. J.C. is an independent statistical consultant.

\section{References}

1. Heeney, D.D.; Gareau, M.G.; Marco, M.L. Intestinal Lactobacillus in health and disease, a driver or just along for the ride? Curr. Opin. Biotechnol. 2018, 49, 140-147. [CrossRef]

2. Arboleya, S.; Watkins, C.; Stanton, C.; Ross, R.P. Gut Bifidobacteria Populations in Human Health and Aging. Front. Microbiol. 2016, 7, 1204. [CrossRef]

3. Gaufin, T.; Tobin, N.H.; Aldrovandi, G.M. The importance of the microbiome in pediatrics and pediatric infectious diseases. Curr. Opin. Pediatr. 2018, 30, 117-124. [CrossRef]

4. McFarland, L.V.; Evans, C.T.; Goldstein, E.J.C. Strain-Specificity and Disease-Specificity of Probiotic Efficacy: A Systematic Review and Meta-Analysis. Front. Med. 2018, 5, 124. [CrossRef] 
5. Naser, S.M.; Hagen, K.E.; Vancanneyt, M.; Cleenwerck, I.; Swings, J.; Tompkins, T.A. Lactobacillus suntoryeus Cachat and Priest 2005 is a later synonym of Lactobacillus helveticus (Orla-Jensen 1919) Bergey et al. 1925 (Approved Lists 1980). Int. J. Syst. Evol. Microbiol. 2006, 56, 355-360. [CrossRef] [PubMed]

6. Zheng, J.; Wittouck, S.; Salvetti, E.; Franz, C.; Harris, H.M.B.; Mattarelli, P.; O’Toole, P.W.; Pot, B.; Vandamme, P.; Walter, J.; et al. A taxonomic note on the genus Lactobacillus: Description of 23 novel genera, emended description of the genus Lactobacillus Beijerinck 1901, and union of Lactobacillaceae and Leuconostocaceae. Int. J. Syst. Evol. Microbiol. 2020, 70, 2782-2858. [CrossRef] [PubMed]

7. Canada Vigilance Adverse Reaction Online Database. Government of Canada. Available online: https://www.canada.ca/en/ health-canada/services/drugs-health-products/medeffect-canada/adverse-reaction-database.html (accessed on 21 May 2021).

8. Balduzzi, S.; Rücker, G.; Schwarzer, G. How to perform a meta-analysis with R: A practical tutorial. Evid. Based Ment. Health 2019, 22, 153-160. [CrossRef] [PubMed]

9. Schwarzer, G. Meta: An R Package for Meta-Analysis. R News 2007, 7, 40-45. Available online: http://cran.r-project.org/doc/ Rnews/Rnews_2007-3.pdf (accessed on 31 May 2021).

10. Higgins, J.P.; Altman, D.G.; Gøtzsche, P.C.; Jüni, P.; Moher, D.; Oxman, A.D.; Savovic, J.; Schulz, K.F.; Weeks, L.; Sterne, J.A. The Cochrane Collaboration's tool for assessing risk of bias in randomised trials. BMJ 2011, 343, d5928. [CrossRef]

11. Duval, S.; Tweedie, R. Trim and fill: A simple funnel-plot-based method of testing and adjusting for publication bias in meta-analysis. Biometrics 2000, 56, 455-463. [CrossRef]

12. Duval, S.; Tweedie, R. A Nonparametric "Trim and Fill” Method of Accounting for Publication Bias in Meta-Analysis. J. Am. Stat. Assoc. 2000, 95, 89-98. [CrossRef]

13. Manzano, S.; De Andres, J.; Castro, I.; Rodriguez, J.M.; Jimenez, E.; Espinosa-Martos, I. Safety and tolerance of three probiotic strains in healthy infants: A multi-centre randomized, double-blind, placebo-controlled trial. Benef. Microbes 2017, 8, 569-578. [CrossRef] [PubMed]

14. Xiao, L.; Gong, C.; Ding, Y.; Ding, G.; Xu, X.; Deng, C.; Ze, X.; Malard, P.; Ben, X. Probiotics maintain intestinal secretory immunoglobulin A levels in healthy formula-fed infants: A randomised, double-blind, placebo-controlled study. Benef. Microbes 2019, 2019, 1-12. [CrossRef] [PubMed]

15. Gao, M. The Efficacy Analysis of the Combination of Smecta and BIOSTIME Probiotics in the Treatment of Non-Infectious Diarrhea in Children. Med. Front. 2013, 164. Available online: https://irp-cdn.multiscreensite.com/a17cd43e/files/uploaded/ Gao-2013-The\%20efficacy\%20analysis\%20of\%20the\%20combinat.pdf (accessed on 31 May 2021).

16. Li, S. Efficacy analysis of Montmorillonite combined with Biostime probiotics in children with non-infectious diarrhea. Spec. Health Issue 2020, 10, 92.

17. Liang, Y.; Wang, X.; Zhao, B. Efficacy analysis of Montmorillonite combined with Biostime probiotics in children with noninfectious diarrhea. Women Health Res. 2018, 121-123. [CrossRef]

18. Liu, P.; Liang, Y.; Zhu, M. Observation on the efficacy of Montmorillonite Powder and Biostime Probiotics in Children with non-infectious diarrhea. Hebei Med. J. 2015, 37, 1846-1847.

19. Luo, G. Smecta Combined with Biostime Probiotics in the Treatment of Non-Infectious Diarrhea in Children. China Rural Health 2013, 311-312. Available online: https:/ /xueshu.baidu.com/usercenter/paper/show?paperid=1d1d04y0fm4r0ja0bd4h0c90vh477 $682 \&$ site $=x u e s h u \_s e($ accessed on 31 May 2021).

20. Wang, F. The effectiveness analysis of combination of Smecta and Biostime probiotics in the treatment of infantile non-infectious diarrhea. Mod. Prev. Med. 2012, 39, 2726-2727.

21. $\mathrm{Wu}, \mathrm{X}$. The efficacy analysis of the combination of Smecta and Biostime probiotics in the treatment of non-infectious diarrhea in children. Road Health 2013, 12, 180-181.

22. Cui, X.; Wure, G. The Treatment of 62 Cases of Rotavirus Gastroenteritis by Biostime Probiotics. Chin. J. Synth. Med. 2003, 5, 53-54.

23. Jin, Y. Clinical Efficacy Observation of Biostime Probiotics in the Treatment of Autumn Diarrhea. Chin. J Clin. Ration. Drug Use 2014, 7, 44-45.

24. Li, X.; Chen, Z. Evaluation of the efficacy of Biostime on rotaviral infection in children. Med. Inf. 2008, 21, 893-895.

25. Yang, J.; Tian, X.; Chen, Y.; Li, P.; Lin, Y.; Wang, Y.; Xu, Z.; Zhao, W. Clinical Observation of Different Feeding Methods in Adjuvant Treatment of Infantile Diarrhea. Occup. Health 2010, 26, 2807-2808.

26. Jiang, X. Clinical Evaluation of Biostime in the Treatment of Children with Persistent Diarrhea. In Proceedings of the 6th Chinese National Conference Pediatric Microecology, Shenzhen, China, 6 November 2016; Available online: https://xueshu.baidu.com/ usercenter/paper/show?paperid=de84a9406f735672673620e40b7869eb\&site=xueshu_se\&hitarticle=1 (accessed on 31 May 2021).

27. Chen, L.; Ouyang, L.; Liao, W.; Zhang, W. Mucous membrane immunity enhanced by taking Biostime probiotics. Chin. J. Ecol. 2007, 19, 137-141.

28. De Andres, J.; Manzano, S.; Garcia, C.; Rodriguez, J.M.; Espinosa-Martos, I.; Jimenez, E. Modulatory effect of three probiotic strains on infants' gut microbial composition and immunological parameters on a placebo-controlled, double-blind, randomised study. Benef. Microbes 2018, 9, 573-584. [CrossRef] [PubMed]

29. Pantović, F. Serum immunoglobulin levels in children with respiratory infections who used a synbiotic dietary supplement. PONS Med. J. 2013, 10, 7-11. [CrossRef] 
30. Qiu, W.; Zhang, F.; Sun, L.; Yu, J.; Wang, S.; Li, G.; Tong, X. The effect of blue light phototherapy combined with synbiotics on neonatal hyperbilirubinemia. Chin. J. Med. 2020, 55, 1014-1016.

31. Cazzola, M.; Pham-Thi, N.; Kerihuel, J.C.; Durand, H.; Bohbot, S. Efficacy of a synbiotic supplementation in the prevention of common winter diseases in children: A randomized, double-blind, placebo-controlled pilot study. Ther. Adv. Respir. Dis. 2010, 4, 271-278. [CrossRef]

32. Stojković, A.; Simović, A.; Bogdanović, Z.; Banković, D.; Poskurica, M. Optimal time period to achieve the effects on synbioticcontrolled wheezing and respiratory infections in young children. Srp. Arh. Celok. Lek. 2016, 144, 38-45. [CrossRef] [PubMed]

33. Xi, Y.; Ding, S.; Guo, J.; Ma, S.; Kong, X.; Pan, L. Observation of Clinical effects of Biostime probiotics on thrush. Chin. J. Microecol. 2013, 25, 830-831.

34. Li, F. Biostime probiotics combined with compound Huangbai solution in treatment of children eczema: An observation on the efficacy. China Pediatrics Integr. Tradit. West. Med. 2017, 9, 121-123.

35. Wei, J. Evaluation of Synbiotics Probiotics Granules and Iron Dextran Oral Liquid in the Treatment of Pediatric IDA. J. Med. Theory Pract. 2018, 31, 566-567.

36. $\mathrm{Wu}, \mathrm{W}$. Prospective study of iron dextran combined with synbiotic probiotics in the treatment of nutritional iron deficiency anemia in children. Matern. Child Health Care China 2014, 29, 3284-3286.

37. Yang, Y. Efficacy analysis of the combined treatment of iron dextran oral liquid and Biostime probiotics in children with nutritional iron deficiency. Matern. Child Health Care China 2015, 30, 6390-6392.

38. Huang, T.; Ouyang, F. Clinical Study on the Effect of Biostime Probiotics Granules on Gastrointestinal Function in Very Low Birth Weight Infants. Med. Inf. 2015, 28, 317-318.

39. Gao, Y.; Peng, F.; Wang, J.; Zhu, W.; Wang, S.; Xu, J.; Liu, P.; Sun, Z. Study on the preventive effect of probiotics on neonatal jaundice and neonatal tolerance of probiotics. Matern. Child Health Care China 2015, 30, 6229-6231.

40. Fang, H.; Duan, S.; Dong, Z.; Yu, A.; Liu, X.; Zhang, J.; Peng, W.; Xiao, D.; Zheng, Q.; Xie, L.; et al. The diagnosis and treatment strategy of diarrhea in China. Chin. J. Pract. Pediatr. 1998, 13, 381-384.

41. Sohail, G.; Xu, X.; Christman, M.C.; Tompkins, T.A. Probiotic Medilac-S ${ }^{\circledR}$ for the induction of clinical remission in a Chinese population with ulcerative colitis: A systematic review and meta-analysis. World J. Clin. Cases 2018, 6, 961-984. [CrossRef] [PubMed]

42. Corthesy, B. Multi-faceted functions of secretory IgA at mucosal surfaces. Front. Immunol. 2013, 4, 185. [CrossRef]

43. Mantis, N.J.; Rol, N.; Corthesy, B. Secretory IgA's complex roles in immunity and mucosal homeostasis in the gut. Mucosal. Immunol. 2011, 4, 603-611. [CrossRef]

44. Cacho, N.T.; Lawrence, R.M. Innate Immunity and Breast Milk. Front. Immunol. 2017, 8. [CrossRef] [PubMed]

45. Lewis, D.B.; Wilson, C.B. CHAPTER 4-Developmental Immunology and Role of Host Defenses in Fetal and Neonatal Susceptibility to Infection. In Infectious Diseases of the Fetus and Newborn, 7th ed.; Remington, J.S., Klein, J.O., Wilson, C.B., Nizet, V., Maldonado, Y.A., Eds.; W.B. Saunders: Philadelphia, PA, USA, 2011; pp. 80-191. [CrossRef]

46. Leong, K.W.; Ding, J.L. The unexplored roles of human serum IgA. DNA Cell Biol. 2014, 33, 823-829. [CrossRef] [PubMed]

47. Yel, L. Selective IgA deficiency. J. Clin. Immunol. 2010, 30, 10-16. [CrossRef] [PubMed]

48. Neonatology Group of Pediatrics Branch of Chinese Medical Association, Editorial Board of Chinese Journal of Pediatrics. Expert consensus on diagnosis and treatment of neonatal hyperbilirubinemia. Chin. Pediatrics J. 2014, 52, 745-748.

49. Van den Nieuwboer, M.; Brummer, R.J.; Guarner, F.; Morelli, L.; Cabana, M.; Claassen, E. Safety of probiotics and synbiotics in children under 18 years of age. Benef. Microbes 2015, 6, 615-630. [CrossRef]

50. Land, M.H.; Rouster-Stevens, K.; Woods, C.R.; Cannon, M.L.; Cnota, J.; Shetty, A.K. Lactobacillus sepsis associated with probiotic therapy. Pediatrics 2005, 115, 178-181. [CrossRef]

51. Salminen, M.K.; Rautelin, H.; Tynkkynen, S.; Poussa, T.; Saxelin, M.; Valtonen, V.; Järvinen, A. Lactobacillus bacteremia, clinical significance, and patient outcome, with special focus on probiotic L. rhamnosus GG. Clin. Infect. Dis. 2004, 38, 62-69. [CrossRef]

52. Higgins, J.P.; Thompson, S.G.; Spiegelhalter, D.J. A re-evaluation of random-effects meta-analysis. J. R. Stat. Soc. Ser. A Stat. Soc. 2009, 172, 137-159. [CrossRef]

53. Culpepper, T.; Christman, M.C.; Nieves, C., Jr.; Specht, G.J.; Rowe, C.C.; Spaiser, S.J.; Ford, A.L.; Dahl, W.J.; Girard, S.A.; Langkamp-Henken, B. Bifidobacterium bifidum R0071 decreases stress-associated diarrhoea-related symptoms and self-reported stress: A secondary analysis of a randomised trial. Benef. Microbes 2016, 7, 327-336. [CrossRef]

54. Langkamp-Henken, B.; Rowe, C.C.; Ford, A.L.; Christman, M.C.; Nieves, C., Jr.; Khouri, L.; Specht, G.J.; Girard, S.A.; Spaiser, S.J.; Dahl, W.J. Bifidobacterium bifidum R0071 results in a greater proportion of healthy days and a lower percentage of academically stressed students reporting a day of cold/flu: A randomised, double-blind, placebo-controlled study. Br. J. Nutr. 2015, 113, 426-434. [CrossRef]

55. Macpherson, C.; Audy, J.; Mathieu, O.; Tompkins, T.A. Multistrain probiotic modulation of intestinal epithelial cells' immune response to a double-stranded RNA ligand, poly(i·c). Appl. Environ. Microbiol. 2014, 80, 1692-1700. [CrossRef]

56. MacPherson, C.W.; Shastri, P.; Mathieu, O.; Tompkins, T.A.; Burguière, P. Genome-Wide Immune Modulation of TLR3-Mediated Inflammation in Intestinal Epithelial Cells Differs between Single and Multi-Strain Probiotic Combination. PLoS ONE 2017, 12, e0169847. [CrossRef] [PubMed] 
57. Kuugbee, E.D.; Shang, X.; Gamallat, Y.; Bamba, D.; Awadasseid, A.; Suliman, M.A.; Zang, S.; Ma, Y.; Chiwala, G.; Xin, Y.; et al. Structural Change in Microbiota by a Probiotic Cocktail Enhances the Gut Barrier and Reduces Cancer via TLR2 Signaling in a Rat Model of Colon Cancer. Dig. Dis. Sci. 2016, 61, 2908-2920. [CrossRef]

58. Cazzola, M.; Tompkins, T.A.; Matera, M.G. Immunomodulatory impact of a synbiotic in $\mathrm{Th}_{1}$ and $\mathrm{Th}_{2}$ models of infection. Ther. Adv. Respir. Dis. 2010, 4, 259-270. [CrossRef] [PubMed]

59. Wang, X.; Yang, J.; Zhang, H.; Yu, J.; Yao, Z. Oral probiotic administration during pregnancy prevents autism-related behaviors in offspring induced by maternal immune activation via anti-inflammation in mice. Autism. Res. 2019, 12, 576-588. [CrossRef]

60. Ng, Q.X.; Loke, W.; Venkatanarayanan, N.; Lim, D.Y.; Soh, A.Y.S.; Yeo, W.S. A Systematic Review of the Role of Prebiotics and Probiotics in Autism Spectrum Disorders. Medicina 2019, 55, 129. [CrossRef] [PubMed]

61. Robinson, S.; Zhu, H.; Zhang, Z.; Li, B.; Pierro, A.; Sherman, P.M. A103 Probiotic Formulations Differentially Affect Disease Outcome in a Mouse Model of Necrotizing Enterocolitis. J. Can. Assoc. Gastroenterol. 2020, 3, 119-120. [CrossRef]

62. Zhang, X.; Li, L.; Butcher, J.; Stintzi, A.; Figeys, D. Advancing functional and translational microbiome research using meta-omics approaches. Microbiome 2019, 7, 154. [CrossRef] [PubMed]

63. Yan, F.; Polk, D.B. Probiotics and Probiotic-Derived Functional Factors-Mechanistic Insights Into Applications for Intestinal Homeostasis. Front. Immunol. 2020, 11. [CrossRef] [PubMed]

64. Paul, W.; Marta, C.; Tom, V.W. Resolving host-microbe interactions in the gut: The promise of in vitro models to complement in vivo research. Curr. Opin. Microbiol. 2018, 44, 28-33. [CrossRef] [PubMed]

65. Cassotta, M.; Forbes-Hernández, T.Y.; Calderón Iglesias, R.; Ruiz, R.; Elexpuru Zabaleta, M.; Giampieri, F.; Battino, M. Links between Nutrition, Infectious Diseases, and Microbiota: Emerging Technologies and Opportunities for Human-Focused Research. Nutrients 2020, 12, 1827. [CrossRef] [PubMed] 